\title{
A STUDY ON GLYCAEMIC STATUS IMPACT IN ACUTE CORONARY SYNDROME
}

\author{
Venkateshwarlu Rajuri' ${ }^{1}$ Priyanka N. ${ }^{2}$
}

${ }^{1}$ Associate Professor, Department of Medicine, Chalmeda Anand Rao Institute of Medical Sciences, Karimnagar, Telangana, India. 2Junior Resident, Department of Medicine, Chalmeda Anand Rao Institute of Medical Sciences, Karimnagar, Telangana, India.

\begin{abstract}
BACKGROUND
Coronary Artery Disease is the leading cause of death worldwide currently, resulting in around 7 million deaths per year and can rise by $40 \%$ around 2020. The prevalence of CAD and the incidence of ACS also are very high and occurs 5-10 years earlier among Indians. The overall cardiovascular mortality is predicted to have risen by $103 \%$ in men and $90 \%$ in women between 1985 and 2015. Hyperglycaemia is an independent risk factor for cardiovascular diseases. Therefore, we evaluated the effect of HbA1c values on clinical outcomes in diabetic and non-diabetic patients following acute coronary syndromes (ACS).
\end{abstract}

ABSTRACT

\section{METHODS}

The study was conducted in patients admitted with acute coronary syndromes in Department of Medicine in Chalmeda Anand Rao Institute of Medical Sciences from January 2017 to September 2018. Chi-square test was used wherever comparisons were needed between the two groups, or between two categories in the same group. A p value $<0.05$ was considered significant.

\section{RESULTS}

SVD was seen in $100 \%$ of patients with $\mathrm{HbA1c}<5 \%$. SVD and DVD were seen in 84.2 and $15.7 \%$ of patients with $\mathrm{HbA} 1 \mathrm{c} 5.1-5.6 \%$, respectively. SVD, DVD and TVD were seen in 57.65\%, 34.6\% and 7.6\% of patients with HbA1c 5.7-6.4\% respectively. SVD, DVD and TVD were seen in $50 \%, 15.3 \%$ and $34.6 \%$ of patients with HbA1c $>6.5 \%$ respectively. In our study, RCA (42) was most frequently affected vessel followed by LAD (37) and LCX (20).

\section{CONCLUSIONS}

Higher HbA1c values were associated with more severe disease and more extensive involvement of coronary vessels in diabetics as well as pre-diabetics. RCA followed by LAD are common culprit vessel in diabetics.

\section{KEY WORDS}

ACS- Acute Coronary Syndrome, HbA1c- Glycosylated Haemoglobin, AGE- Advanced Glycation End Products, Diabetics, Atherosclerosis

HOW TO CITE THIS ARTICLE: Rajuri V, Priyanka N. A study on glycaemic status impact in acute coronary syndrome. J. Evolution Med. Dent. Sci. 2019;8(25):1983-1987, DOI: 10.14260/jemds/2019/436

\section{BACKGROUND}

Coronary Artery Disease is the leading cause of death worldwide currently, resulting in around 7 million deaths per year[1] and can rise by $40 \%$ around 2020 .

The prevalence of CAD and the incidence of ACS also are very high and occurs 5-10 years earlier among Indians.[2] The overall cardiovascular mortality is predicted to have risen by 103\% in men and 90\% in women between 1985 and 2015.[3]

Hyperglycaemia is an independent risk factor for cardiovascular diseases.[4],[5] Therefore, we evaluated the effect of HbA1c values on clinical outcomes in diabetic and non-diabetic patients following acute coronary syndromes (ACS).

In India, there is a joint epidemic of type 2 diabetes and Coronary Artery Disease (CAD).[6] Epidemiological studies in urban and rural population performed over the last 50 years have clearly shown an increase in the prevalence of diabetes as well as CAD in rural and urban population.

'Financial or Other Competing Interest': None.

Submission 26-05-2019, Peer Review 15-06-2019,

Acceptance 17-06-2019, Published 24-06-2019.

Corresponding Author:

Dr. Venkateshwarlu Rajuri,

Associate Professor, Department of Medicine,

Chalmeda Anandrao Institute of Medical Sciences,

Bommakal, Karimnagar, Telangana, India.

E-mail: drvenrajuri@gmail.com

DOI: $10.14260 /$ jemds/2019/436
The prevalence of type 2 diabetes has increased from less than $1 \%$ in rural and $1.5 \%$ in urban areas in 1960 s to $6-8 \%$ in rural and $10-15 \%$ in urban population currently. ${ }^{[7]}$ The prevalence of CAD has increased from less than $1 \%$ in rural populations to $4-5 \%$ presently, and in urban area from $1-2 \%$ in 1960 "s to $9-12 \%$ presently. [7] Diabetes is now considered a cardiovascular disease equivalent by the UN National Cholesterol Education Program Guidelines, with both microvascular and macrovascular complications. It accelerates the process of atherosclerosis through advanced glycation end products. The elevation of glucose could simply be a marker of pre-existing but not yet detected type 2 diabetes or impaired glucose tolerance ${ }^{[8]}$ or may represent stress hyperglycaemia.[9] Glycosylated haemoglobin (HbA1c) provides a measure of average blood glucose levels during a period of 8-12 weeks (2- 3 months).[10] HbA1c is a good marker of glycated proteins and is an excellent predictor of long-term complications. ${ }^{[4]}$

Elevated HbA1c is associated with poorer prognosis in patients with $\mathrm{LVEF}<45 \%$.[11] $^{[1}$ Even in non-diabetics with $\mathrm{HbA} 1 \mathrm{c} 5 \%$ to $6.4 \%$ is associated with cardiovascular disease and all-cause mortality. Both plasma glucose levels and glycosylated haemoglobin (HbA1c) were implicated as predictors of prognosis.

So, Glycosylated haemoglobin could prove useful for the diagnosis of diabetes mellitus in acute myocardial infarction when stress induced hyperglycaemia is a confounding factor as HbA1c is unlikely to rise with acute hyperglycaemia.[12] 


\section{Aims and Objectives}

1. To study HbA1c as a prognostic marker in acute coronary syndromes in both diabetic and non-diabetic patients.

2. To assess the complications and outcomes among different groups in relation to glycaemic status.

\section{METHODS}

\section{Study Design}

Cross-sectional observational study.

\section{Sample Size}

In our study, we included 74 cases of acute coronary syndrome, who had presented within 48 hours of onset of symptoms, irrespective of diabetic status and who satisfied the inclusion criteria after obtaining informed consent and approval from Institute Ethical Committee. Sample size was taken based on the convenience of the study.

\section{Inclusion Criteria}

Patients with STEMI, NSTEMI and unstable angina who underwent coronary angiogram.

\section{Exclusion Criteria}

Patients with haemoglobinopathy, hypothyroidism, renal failure and sepsis were excluded from the study. Patients in whom HbA1c could not be done were also excluded from the study.

After a detailed history and physical examination, admission glucose, HbA1c, lipid profile, cardiac enzymes, ECG and Echocardiogram were done in all patients. Coronary angiography findings were noted in all patients. Treatment was given as per standard protocol (American College of Cardiology guidelines) in the ICCU.

The patients were followed up till discharge and all complications like arrhythmias, cardiac failure, cardiogenic shock and death were noted. HbA1c levels were estimated on Diastat from BioRad. Patients taken were categorized into four groups based on their HbA1c values.

Complications and outcomes were compared in all four groups.

Group 1: HbA1c <5\% (Lower range in non-diabetic group)

Group 2: HbA1c 5.1-5.6\% (Higher range in non-diabetic group)

Group 3: HbA1c 5.7-6.4\% (Pre diabetic range)

Group 4: HbA1c $>6.5 \%$ (Diabetic range)

\section{Statistical Analysis}

Statistical analyses were carried out using a computer based statistical analysis programme, SPSS (Statistical Program for Social Sciences) version 11.5. The Chi-square test was used wherever comparisons were needed between the two groups, or between two categories in the same group. A p value < 0.05 was considered significant.

\section{RESULTS}

The analysis of data is as follows.

HbA1c $<5 \%$ was seen in $4.1 \%$ of the patients, between $5.1-5.6 \%$ in $25.7 \%$, between $5.7-6.4 \%$ in $35.1 \%$, and $>6.5 \%$ in $35.1 \%$ of patients.

Males were $66 \%$ and females were $33 \%$ in $\mathrm{HbA} 1 \mathrm{c}<5 \%$ group. Males were $84.2 \%$ and females were $15.7 \%$ in $\mathrm{HbA} 1 \mathrm{c}$
5.1-5.6\% group. Males were $69.2 \%$ and females were 30.7 in HbA1c 5.7-6.4\% group. Males were $65.3 \%$ and females were $34.6 \%$ in $\mathrm{HbA} 1 \mathrm{c}>6.5 \%$ group.

Mean age (years) of the patient was 54.6(8.7) in HbA1c $<5 \%, 57.7(14.3)$ in $\mathrm{HbA1c}$ between $5.1-5.6 \%, 55.9(13)$ in $\mathrm{HbA} 1 \mathrm{c}$ between 5.7-6.4\%, and 59.8(8.9) in $\mathrm{HbA} 1 \mathrm{c}>6.5 \%$.

UA, NSTEMI, and STEMI was seen in 33\%, 33\%, and 33\% of patients with $\mathrm{HbA} 1 \mathrm{c}<5 \%$ respectively. UA, NSTEMI, and STEMI was seen in $31 \%, 36.8 \%$, and $31.58 \%$ of patients with HbA1c 5.1-5.6\% respectively. UA, NSTEMI, and STEMI was seen in $38 \%, 30.7 \%$, and $30.7 \%$ of patients with HbA1c 5.7$6.4 \%$ respectively. UA, NSTEMI and STEMI was seen in $15 \%$, $65 \%$, and $19 \%$ patients with $\mathrm{HbA} 1 \mathrm{c}>6.5 \%$ respectively.

Out of 74 patients, 35 patients had hypertension. History of hypertension was seen in $5.7 \%$ of patients with $\mathrm{HbA} 1 \mathrm{c}$ $<5 \%, 14.3 \%$ of patients with HbA1c $5.1-5.6 \%, 40 \%$ of patients with $\mathrm{HbA1c} 5.7-6.4 \%$ and $40 \%$ of patients with HbA1c $>6.5 \%$

$66 \%$ of patients with $\mathrm{HbA} 1 \mathrm{c}<5 \%$ were smokers, $21 \%$ of patients with $\mathrm{HbA1c} 5.1-5.6 \%, 15.3 \%$ of patients with $\mathrm{HbA} 1 \mathrm{c}$ $5.7-6.4 \%$ and $30.7 \%$ of patients with $\mathrm{HbA} 1 \mathrm{c}>6.5 \%$ were smokers. $10.5 \%$ of patients with HbA1c $5.1-5.6 \%, 3.8 \%$ of patients with $\mathrm{HbA1c} 5.7-6.4 \%$, and $7.6 \%$ of patients with HbA1c $>6.5 \%$ had history of alcohol consumption.

Consumption of tobacco and alcohol was present in 33\% of patients with $\mathrm{HbA1c}<5 \%, 36 \%$ of patients with $\mathrm{HbA} 1 \mathrm{c} 5.1$ $5.6 \%, 19 \%$ of patients with HbA1c $5.7-6.4 \%$, and $15 \%$ of patients with $\mathrm{HbA} 1 \mathrm{c}>6.5 \%$.

Out of 74 patients, 20 patients had STEMI and 54 patients had NSTEMI/UA. In patients with STEMI, 65\% patients had history of active smoking, 38.8\% patients of NSTEMI/UA had history of active smoking. There is ( $p, 0.0453)$ significant association between active smoking and STEMI.

Normal BMI was seen in $100 \%$ of patients with HbA1c $<5 \%, 94 \%$ of patients with $\mathrm{HbA} 1 \mathrm{c} 5.1-5.6 \%, 73 \%$ of patients with $\mathrm{HbA} 1 \mathrm{c} 5.7-6.4 \%$ and $53 \%$ of patients with $\mathrm{HbA} 1 \mathrm{c}>6.5 \%$. $5 \%$ of patients with $\mathrm{HbA1c} 5.1-5.6 \%, 23 \%$ of patients with $\mathrm{HbA} 1 \mathrm{c} 5.7-6.4 \%$ and $38 \%$ of patients with $\mathrm{HbA} 1 \mathrm{c}>6.5 \%$ were overweight. $3 \%$ of patients with HbA1c 5.7-6.4, and $7.6 \%$ of patients with $\mathrm{HbA} 1 \mathrm{c}>6.5 \%$ were obese.

Dyslipidaemia was seen in $33 \%$ of patients with $\mathrm{HbA1c}$ $<5 \%, 73.6 \%$ of patients with $\mathrm{HbA} 1 \mathrm{c} 5.1-5.6 \%, 65.3 \%$ of patients with HbA1c $5.7-6.4 \%$ and $88.4 \%$ of patients with HbA1c $>6.5 \%$. There was no significant association between dyslipidaemia and $\mathrm{HbA1c}$.

Mean baseline EF (\%) was 47.6(7.3) in $\mathrm{HbA1c}<5 \%$, 48.4(7.5) in HbA1c between 5.1-5.6\%, 45.8(6.1) in HbA1c between $5.7-6.4 \%$, and $44.4(6.1)$ in $\mathrm{HbA1c}>6.5 \%$. EF did not vary significantly among groups, but mean LVEF $<45 \%$ was found in diabetic group.

SVD was seen in $100 \%$ of patients with $\mathrm{HbA} 1 \mathrm{c}<5 \%$. SVD and DVD were seen in 84.2 and $15.7 \%$ of patients with $\mathrm{HbA} 1 \mathrm{c}$ 5.1-5.6\%, respectively. SVD, DVD and TVD were seen in $57.65 \%, 34.6 \%$ and $7.6 \%$ of patients with $\mathrm{HbA} 1 \mathrm{c} 5.7-6.4 \%$ respectively. SVD, DVD and TVD were seen in 50\%, 15.3\% and $34.6 \%$ of patients with $\mathrm{HbA} 1 \mathrm{c}>6.5 \%$ respectively. This was found to have a significant association. Multiple vessels were involved in patients with $\mathrm{HbA1c}$ in prediabetic and diabetic range of $\mathrm{HbA} 1 \mathrm{c}$.

In our study, RCA (42) was most frequently affected vessel followed by LAD (37) and LCX (20). Out of 11 patients with TVD, 8 (72.7\%) patients had involvement of LAD, LCX and RCA. Among these 8 patients, 7 patients had HbA1c in 
diabetic range of $>6.5$ and 1 patient had $\mathrm{HbA} 1 \mathrm{c}$ in prediabetic range.

In our study, 53 (71.6\%) patients had STEMI and NSTEMI. In these patients, 46 (86.7\%) patients had IWMI. 7 (13.3\%) patients had of anterior or lateral wall. As RCA was the commonly involved vessel, IWMI was the most common presentation. After RCA, the commonly involved vessel was LAD; these include patients with DVD, TVD and patients with UA who underwent CAG. In patients with $\mathrm{HbA} 1 \mathrm{c}>6.5,69 \%$ of patients had LAD involved. In patients with HbA1c 5\%-6.4\% had LAD involved in 39\% of patients. There was significant correlation between involvement of LAD and diabetic status ( $p$ value $<0.05$ ). Incidence of complications were higher in patients with $\mathrm{HbA1c}$ range of $5.1-5.6 \%$ and $\mathrm{HbA1c}$ range $>6.5 \%$. Patients who had higher $\mathrm{HbA} 1 \mathrm{c}>6.5 \%$ had higher incidence of Cardiogenic shock compared to patients with HbA1c $<6.4 \%$. (p value $<0.05$ ).

\section{DISCUSSION}

A comparative analysis of those with a previous history of diabetes and those without diabetes was done initially. Based on $\mathrm{HbA1c}$ values patients were further categorized into four groups:

In our study out of 74 patients $3(4.1 \%)$ patients were in range of $\mathrm{HbA1c}<5 \%$ [Group 1], 19 (25.7\%) patients were in the range of $\mathrm{HbA1c} 5.1-5.6 \%$ [Group 2], 26 (35.1\%) were in the range of HbA1c 5.7-6.4\% [Group 3] and 26 (35.1\%) patients were in the range of $\mathrm{HbA} 1 \mathrm{c}>6.5 \%$ [Group 4].

\section{Gender and HbA1c}

In our study, out of 74 patients 53 were male and 21 were female patients. There was no statistically significant correlation between gender and HbA1c ( $p$ value 0.55 ) in our study although a study by Tingting $\mathrm{Du}^{[13]}$ documents that HbA1c-defined non-diabetic men tended to have more favourable CVD risk profile than women. More CVD risk factors were observed in $\mathrm{HbA} 1 \mathrm{c}$ defined diabetic subjects, irrespective of gender. According to their study, men who progressed from HbA1c-defined non-diabetes to HbA1cdefined diabetes have greater deterioration in many CVD risk factors.

\section{Age and HbA1c}

In our study no significant correlation between Hba1c and mean age of presentation in four groups. (p value 0.67) Studies done by Naseem et al[14] showed diabetic patients were susceptible to ACS at an earlier age compared to nondiabetics.

\section{Past History of IHD and HbA1c}

In our study only 3 patients had past history of ischemic heart disease and all 3 patients had history of diabetes and had $\mathrm{HbA} 1 \mathrm{c}>6.5 \%$. In our study due to limited number of subjects, this association could not be analysed.

\section{Hypertension and ACS}

In our study 35 out of 74 patients had past history of hypertension. Among these patients $80 \%$ of patients had $\mathrm{HbA1c}$ in pre-diabetic and diabetic range (Group 3 and group 4). There was no significant correlation between HbA1c and hypertension ( $\mathrm{p}$ value 0.195 ).

Several studies reported that a history of hypertension was associated with an increased rate of adverse outcomes after AMI such as stroke, heart failure and cardiovascular death.[15]

\section{Obesity and HbA1c}

In our study $3(4 \%)$ out of 74 patients were obese and 17 (22\%) were overweight based on BMI $(\mathrm{kg} / \mathrm{m} 2)$. Remaining $54(72.9 \%)$ patients were having BMI in normal range. 12 out of 26 patients in diabetic range of $\mathrm{HbA1c}$ were overweight (38\%) or obese (7.6\%). 7 out of 26 patients were overweight $(23 \%)$ or obese $(3.8 \%)$ in prediabetes group. This association was statistically not significant. ( $\mathrm{p}$ value 0.102 )

\section{Smoking and Alcohol as Risk Factors in ACS}

In our study there was no significant correlation between smoking and alcohol consumption with HbA1c ( $p$ value 0.34 ). $14(53.8 \%)$ of 26 patients in diabetic range of HbA1c gave history of consumption of alcohol or smoking tobacco.

In our study there was significant association between active smoking and incidence of STEMI ( $p$ value 0.04 ). Certain studies concluded in patients with type 2 diabetes, moderate alcohol use, particularly wine consumption, is associated with reduced risks of cardiovascular events and all-cause mortality.

\section{Dyslipidaemia and HbA1c}

In our study, out of 74 patients included 55 patients (74.3\%) had dyslipidaemia. $23(88.6 \%)$ of 26 patients with $\mathrm{HbA1c}$ $>6.5 \%$ had dyslipidaemia. $17(65.3 \%)$ of 26 patients with HbA1c 5.7-6.4\%, 14 (73.6\%) of 19 patients with HbA1c 5.1$5.6 \%$ and 1 (33.3\%) of 3 patients with $\mathrm{HbA1c}<5 \%$ had dyslipidaemia. This association between dyslipidaemia and HbA1c was statistically not significant. (P- 0.09). Patients with dyslipidaemia who are diabetic are at an elevated risk for adverse cardiovascular outcomes compared with controls. Although in nondiabetic individual LDL is predictive of cardiovascular outcomes, the prevalence of LDL is similar between individuals with diabetes and nondiabetic individuals and data suggest that the elevated risk for CVD in individuals with diabetes occurs independently of the serum LDL level.[16]

\section{Incidence of ACS with HbA1c}

In our study there was no significant correlation between HbA1c and clinical presentation of ACS (STEMI, NSTEMI or UA).

Bogaty et al[17] and Safiuddin et al[18] studies showed chronic stable angina in diabetics and ACS in non-diabetics as the main clinical presentation but no significant difference of STEMI and UA/NSTEMI in diabetic versus non diabetic patients.

\section{Admission Glucose and HbA1c in ACS}

In our study the mean admission glucose was 144.7 (+/- 12.1 SD). There was statistically significant association between HbA1c and admission hyperglycaemia. this could be due to underlying undiagnosed diabetes or as a consequence of stress (Stress hyperglycaemia).

In another study ${ }^{[19]}$ conducted in 100 patients with no previous history of diabetes with ACS to demonstrate the correlation between stress hyperglycaemia and cardiovascular outcome of heart failure, arrhythmias, cardiogenic shock or death. There was high prevalence (38\%) of stress hyperglycaemia in ACS patients even among non- 
diabetics when compared to normoglycemic group had higher incidence of MACE.

\section{HbA1c and Mean LVEF}

In our study we measured LVEF at the time of discharge, and compared among various groups. The mean LVEF was $47.6 \%$, $48.4 \%, 45.8 \%$ and $44.4 \%$ in patients with HbA1c of $<5 \%, 5.1-$ $5.6 \%, 5.7-6.4 \%$ and $>6.5 \%$ respectively. In our study LVEF had no significant association with HbA1c, (P-0.21) although mean LVEF was comparatively lower in patients with $\mathrm{HbA1c}$ $>6.5 \%$.

Goode et al concluded in their study that patients with elevated HbA1c had poorer prognosis in patients with LVEF $<45 \%$. Contrary to our findings, a study done by Muhammad Uwais Ashraf et al[20] in 200 patients with no history of diabetes admitted with ACS showed as linear correlation between ejection fraction and HbA1c ( $p$ value $=0.019$ ). They concluded that $\mathrm{HbA1c}$ was a strong predictor of left ventricular dysfunction in non-diabetic patients presenting with acute coronary syndrome, therefore, measurement of HbA1c levels may improve risk assessment in patients presenting with acute coronary syndrome. Presence of lower $\mathrm{EF}$ indicates development of left ventricular systolic dysfunction in long term.

\section{HbA1c and Severity of CAD}

In our study there was a statistically significant correlation between HbA1c and severity of CAD. ( $p$ value <0.008). Patients with $\mathrm{HbA1c}$ in diabetic range had more extensive disease compared to non-diabetic patients. Another observation was patients with $\mathrm{HbA1c}$ in prediabetic range also had extensive involvement with DVD and TVD compared to patients in non-diabetic range groups. This could be explained by the fact that $\mathrm{HbA1c}$ is an indicator of chronic hyperglycaemia which is a risk factor for development of atherosclerosis.

\section{Association Between Vessels Involved and HbA1c}

In our study the most commonly involved vessel was RCA followed by LAD and LCX. Out of 74 patients, RCA was involved in 42 patients (56.7\%). LAD involved in 37 (50\%) of patients and LCX (27\%) in 20 patients. As mentioned above patients with $\mathrm{HbA} 1 \mathrm{c}>6.5 \%$ had higher incidence of TVD. Out of 11 patients TVD, all patients had HbA1c in pre-diabetic and diabetic range. Three vessels involved in TVD were LAD, RCA and LCX (72.7\%).

In our study, $53(71.6 \%)$ patients had STEMI and NSTEMI. In these patients, $46(86.7 \%)$ patients had IWMI. 7 (13.3\%) patients had of anterior or lateral wall MI. Those patients with anteroinferior and inferolateral MI were also grouped under patients with IWMI. IWMI was grouped separately as patients were prone to shock and arrhythmias more compared to other groups. As RCA was the commonly involved vessel, IWMI was the most common presentation. After RCA, the commonly involved vessel was LAD; these include patients with DVD, TVD and patients with UA who underwent CAG. In patients with $\mathrm{HbA1c}>6.5,69 \%$ of patients had LAD involved. In patients with HbA1c 5\%-6.4\% had LAD involved in $39 \%$ of patients. There was significant correlation between involvement of LAD and diabetic status ( $p$ value $<0.05$ ).
In a study by Tanjima Parvin et al[21]102 patients who had CAD and underwent coronary angiography were studied. In their study Left main stem disease ( $21 \%$ versus $5 \%$, p 0.031$)$ and three-vessel disease (50\% versus $31 \%$, p 0.094) were more prevalent in diabetic patients. The commonest vessel involved in both the groups was LAD, $87 \%$ in diabetic and $71 \%$ in non-diabetic, followed by RCA and LCX. Severe coronary artery stenosis was significantly more in diabetics than non-diabetics. They concluded that diabetic patients are more likely to have severe and extensive coronary artery disease. Left main stem and triple vessel disease are more commonly seen in diabetic patients compared to non-diabetic patients.

\section{HbA1c and MACE}

In our study major adverse cardiac outcomes seen were cardiogenic shock, ventricular arrhythmias and death. Out of 74 patients, 15 patients had cardiogenic shock. Among these 15 patients, $10(66.6 \%)$ patients had $\mathrm{HbA1c}>6.5 \%$. 3 patients had ventricular arrhythmias and there was no association with HbA1c. 4 patients died after coronary revascularization, and there was no specific correlation with HbA1c. Overall, combined incidence of MACE and its association with HbA1c was statistically not significant. ( $p$ value 0.46 )

Cardiogenic shock was the most common complication in patients in our study. The incidence of cardiogenic shock was more in diabetic then in non-diabetic patients. There was a significant correlation between $\mathrm{HbA} 1 \mathrm{c}$ and cardiogenic shock ( $\mathrm{p}$ value 0.004 ).

\section{Limitations}

Our study is done in limited period hence included the relatively small number of event cases of ACS and a smaller sample size.

\section{CONCLUSIONS}

In our study, higher HbA1c values were associated with more severe disease and more extensive involvement of coronary vessels in diabetics as well as pre-diabetics. The RCA followed by LAD are common culprit vessels in diabetics. Patients with higher HbA1c underwent PTCA and CABG more frequently compared to patients with lower HbA1c. In STEMI, smoking has more importance as an independent risk factor. Patients with $\mathrm{HbA} 1 \mathrm{c}>6.5 \%$ had mean $\mathrm{EF}<45 \%$ at the time of discharge compared to other patients, but $p$ value was not significant. Cardiogenic shock as MACE was prominently noted in diabetic group when compared to non-diabetic group. In conclusion, regular estimation of $\mathrm{HbA1c}$ in all patients with ACS is essential for predicting severity and complications.

\section{REFERENCES}

[1] World Health organization (2013). Retrieved from WHO media center, fact sheet: 2013.

[2] Tan AT, Emmanuel SC, Tan BY, et al. Myocardial infarction in Singapore: a nationwide 10-year study of multiethnic differences in incidence and mortality. Ann Acad Med Singapore 2002;31(4):479-86.

[3] Cho NH, Whiting D, Forouhi N, et al. IDF Diabetes Atlas. $7^{\text {th }}$ edn. Brussels, Belgium: International Diabetes Federation 2015: p. 50-2. 
[4] Naidu YR, Sharmila Y, Nischal Y, et al. Study of HbA1c levels in acute coronary syndrome. Journal of Evolution of Medical and Dental Sciences 2015;4(77):13274-8.

[5] Timmer JR, Van der Horst IC, Ottervanger JP, et al. Prognostic value of admission glucose in non-diabetic patients with myocardial infarction. AM Heart J 2004;148(3):399-404.

[6] Mohan V, Sandeep S, Deepa R, et al. Epidemiology of type 2 diabetes: Indian scenario Indian $\mathrm{J}$ Med Res 2007;125(3):217-30.

[7] Gupta R, Kumar P. Global Diabetes landscape - type 2 diabetes mellitus in South Asia: epidemiology, risk factors and control. Insulin 2008;3(2):78-94.

[8] Norhammar A, Tenerz A, Nilsson G, et al. Glucose metabolism in patients with acute myocardial infarction and no previous diagnosis of diabetes mellitus: a prospective study. Lancet 2002;359(9324):2140-4.

[9] Husband DJ, Alberti KG, Julian DG. Stress hyperglycaemia during acute myocardial infarction: an indicator of pre-existing diabetes? Lancet 1983;2(8343):179-81.

[10] American Diabetes Association. Standards of medical care in diabetes (position statement). Diabetes care 2004;27(Suppl 1):S15-S35.

[11] Goode KM, John J, Rigby AS, et al. Elevated glycated haemoglobin is a strong predictor of mortality in patients with left ventricular systolic dysfunction who are not receiving treatment for diabetes mellitus. Heart 2009;95(11):917-23.

[12] Remyasri. HbA1c as a predictor of prognosis following acute coronary syndrome (ACS) and ST elevation MI (STEMI) compared to fasting and admission glucose. WJPMR 2017;3(1):358-88.
[13] Du T, Yuan G, Zhou X, Sex differences in the effect of HbA1c-defined diabetes on a wide range of cardiovascular disease risk factors. Ann Med 2016;48(1-2):34-41.

[14] Naseem A, Moin S. Comparison of Diabetics and Non diabetics presenting with acute myocardial infarction. J Coll Physicians Surg Pak 2002;12(6):356-60.

[15] Thune JJ, Signorovitch J, Kober L, et al. Effect of antecedent hypertension and follow-up blood pressure on outcomes after high-risk myocardial infarction. Hypertension 2008;51(1):48-54.

[16] Haider WJ, Jamal RS. Dyslipidaemia in diabetes mellitus and cardiovascular disease. Cardiovascular Endocrinology \& Metabolism 2017;6(1):27-32.

[17] Bogaty P, Brecker SJ, White SE, et al. Comparison of coronary angiographic findings in acute and chronic first presentation of ischemic heart disease. Circulation 1993;87(6):1938-46.

[18] Shafiuddin M. Coronary angiographic comparison between unheralded acute myocardial infarction and uncomplicated stable angina pectoris. MD thesis, Dhaka University, Bangladesh, 1998.

[19] Aggarwal RC, Goyal M, Sikri H, et al. Prognostic value of stress hyperglycaemia in acute coronary syndrome in non-diabetic patients. Int J Curr Res Med Sci 2017;3(4):7-13.

[20] Ashraf MU, Zaheer MS. Impact of HbA1c on outcomes of acute coronary syndrome in non-diabetic patients. Synergy Publishers 2014. DOI: http://dx doi.org/10.12970/2311-052X.2014.02.03.3

[21] Parvin T, Haque K, Siddique MA, et al. Angiographic severity of coronary artery disease in diabetic and non-diabetic patients in a tertiary care centre. University Heart Journal 2014;10(1):13-7. 